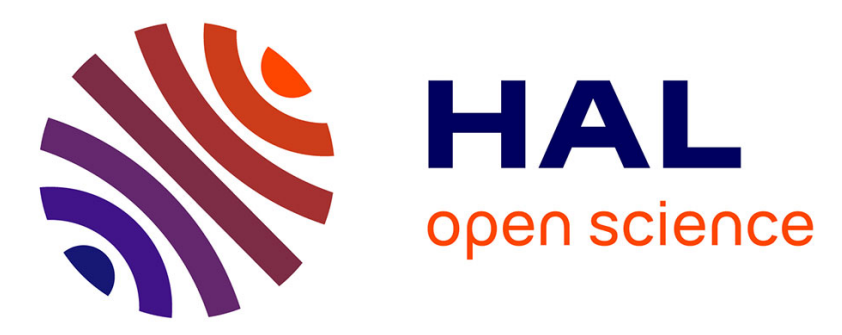

\title{
Analytical and numerical investigations of noncollinear magnetic ordering in the frustrated delafossite $\mathrm{CuCrO} 2$
}

Ahmed Albaalbaky, Yaroslav Kvashnin, Renaud Patte, Denis Ledue

\section{To cite this version:}

Ahmed Albaalbaky, Yaroslav Kvashnin, Renaud Patte, Denis Ledue. Analytical and numerical investigations of noncollinear magnetic ordering in the frustrated delafossite $\mathrm{CuCrO} 2$. Physical Review B: Condensed Matter and Materials Physics (1998-2015), 2019, 99 (10), 10.1103/PhysRevB.99.104415 . hal-02106761

\section{HAL Id: hal-02106761 \\ https://hal-normandie-univ.archives-ouvertes.fr/hal-02106761}

Submitted on 23 Apr 2019

HAL is a multi-disciplinary open access archive for the deposit and dissemination of scientific research documents, whether they are published or not. The documents may come from teaching and research institutions in France or abroad, or from public or private research centers.
L'archive ouverte pluridisciplinaire HAL, est destinée au dépôt et à la diffusion de documents scientifiques de niveau recherche, publiés ou non, émanant des établissements d'enseignement et de recherche français ou étrangers, des laboratoires publics ou privés. 


\title{
Analytical and numerical investigations of noncollinear magnetic ordering in the frustrated delafossite $\mathrm{CuCrO}_{2}$
}

\author{
Ahmed Albaalbaky, ${ }^{1, *}$ Yaroslav Kvashnin, ${ }^{2}$ Renaud Patte,,${ }^{1}$ and Denis Ledue ${ }^{1}$ \\ ${ }^{1}$ Normandie Université, UNIROUEN, INSA Rouen, CNRS, GPM, F-76800 Saint Étienne du Rouvray, France \\ ${ }^{2}$ Department of Physics and Astronomy, Division of Materials Theory, Uppsala University, Box 516, SE-75120 Uppsala, Sweden
}

(Received 9 November 2018; revised manuscript received 25 February 2019; published 12 March 2019)

\begin{abstract}
The magnetic propagation vector in delafossite $\mathrm{CuCrO}_{2}$ with classical Heisenberg spins is calculated analytically as a function of exchange interactions up to fourth-nearest neighbors. Exchange interactions are estimated by a series of density functional theory calculations for several values of lattice distortion. Our calculations show that the magnetic propagation vector is directly affected by the considered distortions providing different stable commensurate or incommensurate magnetic configurations. A realistic set of exchange interactions corresponding to a $0.1 \%$ lattice distortion yields the experimental ground state with an incommensurate propagation vector $\mathbf{q} \sim(0.329,0.329,0)$. We find that a very weak antiferromagnetic interlayer interaction favors an incommensurate ordering even in the absence of lattice distortion. Moreover, the exchange energy of a magnetic configuration of a finite crystal of $\mathrm{CuCrO}_{2}$ with periodic boundary conditions is derived analytically. Based on that, highly accurate Monte Carlo simulations performed on $\mathrm{CuCrO}_{2}$ confirm both the proposed analytical calculations and the density functional theory estimations, where we obtain excellent convergence toward the experimental ground state with a magnetic propagation vector $\mathbf{q}=(0.3288,0.3288,0)$.
\end{abstract}

DOI: 10.1103/PhysRevB.99.104415

\section{INTRODUCTION}

The Heisenberg antiferromagnet on a triangular lattice is one of the prototype examples of frustrated magnetic systems which have been studied for several decades [1-4]. The topology of these systems imposes high geometric magnetic frustration, resulting in several novel phenomena. Most frustrated systems possess the so-called noncollinear spin configurations. Such noncollinear spin ordering breaks the space inversion symmetry, leading to the appearance of new phases, such as ferroelectric ones, in the magnetically ordered state. Delafossite oxides [5-9] are very good examples of frustrated triangular antiferromagnets because they are formed of triangular layers stacked rhombohedrally in a sequence of $A B C A B C \cdots$ type along the vertical direction. Among these delafossite oxides, $\mathrm{CuCrO}_{2}(R \overline{3} \mathrm{~m}$ space group $)$ attracts a lot of attention due to its strong magnetoelectric coupling observed in the magnetically ordered state [10-12]. Also, an interesting novel phenomenon derived from geometric magnetic frustration is the very rich $H-T$ phase diagram obtained in $\mathrm{CuCrO}_{2}$ with several magnetic and ferroelectric phases [13-16]. At room temperature, the triangular layers of $\mathrm{CuCrO}_{2}$ are formed of equilateral triangles stacked in $\mathrm{Cr}^{3+}-\mathrm{O}^{2-}-\mathrm{Cu}^{+}-\mathrm{O}^{2-}-\mathrm{Cr}^{3+}$ layer coordination. Upon a phase transition to a noncollinear antiferromagnetic state at Néel temperature $T_{N}=24-26 \mathrm{~K}$ [17-20], the equilateral triangular layers undergo a small in-plane deformation [21,22] due to a spiral magnetic ordering. Such a lattice deformation is described as a tiny distortion $d$ along the [110] direction leading to anisotropic

*ahmed.baalbaky@hotmail.com first-nearest-neighbor couplings $J_{1} \neq J_{1}^{\prime}$ (Fig. 1) as well as inducing a hard-axis anisotropy along the distorted direction [23]. As a result, an incommensurate noncollinear magnetic ordering with a propagation vector $\mathbf{q}=(0.329,0.329,0)$ pointing along the [110] direction is stabilized below $T_{N}$ [20]. In addition, a spontaneous ferroelectric polarization emerges below $T_{N}$ along the distorted direction due to the variation of the hybridization between $\mathrm{Cr}^{3+} 3 d$ and $\mathrm{O}^{2-} 2 p$ orbitals caused by the spin-orbit coupling [24]. Recent $\mathrm{x}$-ray diffraction measurements [21] performed in $\mathrm{CuCrO}_{2}$ predicted that such a lattice distortion is of the order of $0.01 \%$ at $5 \mathrm{~K}$. In our previous study [23], we have estimated exchange interactions corresponding to $d=0.01 \%$ at $0 \mathrm{~K}$ using density functional theory (DFT) calculations. We found that $J_{1} / J_{1}^{\prime}=0.995$ is not able to reproduce the experimental ground state (GS) in a finite system, and it does not induce a hard-axis anisotropy along the distorted direction necessary for determining a unique spiral plane. For that we arbitrarily multiplied $d$ by a factor of 30 in order to obtain the hard-axis anisotropy responsible for the description of ferroelectric properties in $\mathrm{CuCrO}_{2}$. Such an artificial enhancement of $d$ allowed us to describe magnetoelectric properties at finite temperatures in pristine $\mathrm{CuCrO}_{2}$ [23] as well as $\mathrm{Ga}$-doped $\mathrm{CuCrO}_{2}$ [25] in comparison with experimental observations. In this paper, we focus on the noncollinear magnetic ordering inside the spiral plane of delafossite $\mathrm{CuCrO}_{2}$ to provide a theoretical estimate of $d$ at the GS which leads to the $\mathbf{q}=(0.329$, $0.329,0)$ configuration within the framework of the classical Heisenberg model. For this reason, we derive an analytical expression of $\mathbf{q}=(k, k, 0)$ as a function of the different competing interactions in rhombohedrally stacked distorted triangular lattices. Exchange interactions are estimated 

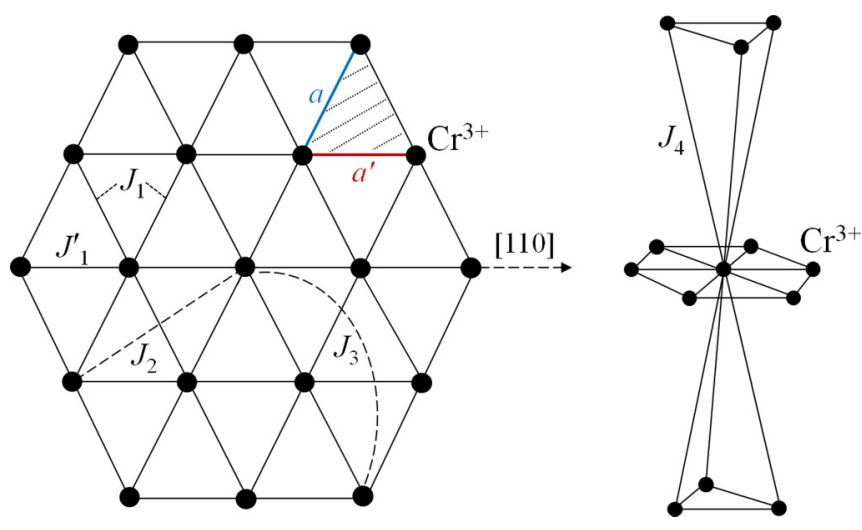

FIG. 1. Distorted triangular lattice $\left(a^{\prime}<a\right)$ of $\mathrm{Cr}^{3+}$ ions with exchange interactions up to fourth-nearest neighbors $\left(J_{1}^{\prime}>J_{1}\right)$ in $\mathrm{CuCrO}_{2}$.

using DFT calculations for various distortions, and the influence of the next-nearest-neighbor interactions on the GS is discussed.

The remainder of this paper is organized as follows: Sec. II presents the derived analytical expression of the propagation vector as a function of exchange interactions. Section III is devoted to discussions of the obtained results, and finally, a conclusion is given in Sec. IV.

\section{ANALYTICAL EXPRESSION OF THE PROPAGATION VECTOR}

Analytical investigations of noncollinear magnetic ordering in three-dimensional (3D) systems with competing interactions are still rarely achieved. Thus, in order to characterize the noncollinear GS configuration in rhombohedrally stacked distorted triangular lattices, we consider the following classical Heisenberg Hamiltonian:

$$
H=-\frac{1}{2} \sum_{i, j} J_{i j} \mathbf{S}_{i} \cdot \mathbf{S}_{j}
$$

where $J_{i j}$ stands for exchange interactions between the interacting spins $\mathbf{S}_{i}$ and $\mathbf{S}_{j}$ up to fourth-nearest neighbors (Fig. 1). Based on Eq. (1), the exchange energy per spin of a magnetic configuration of a propagation vector $\mathbf{q}=(k, k, 0)$ in the distorted infinite crystal is written as

$$
\begin{aligned}
E_{\mathrm{ex}}^{\infty}(k)= & -S^{2}\left[J_{1}^{\prime} \cos (4 \pi k)+2 J_{1} \cos (2 \pi k)\right. \\
& +J_{2}+2 J_{2} \cos (6 \pi k)+J_{3} \cos (8 \pi k) \\
& \left.+2 J_{3} \cos (4 \pi k) \pm J_{4} \pm 2 J_{4} \cos (2 \pi k)\right],
\end{aligned}
$$

where the \pm sign before the $J_{4}$ terms depends on the stable configuration chosen by the system (see Sec. A 1).

Taking a derivative of Eq. (2) with respect to $k$ gives

$$
\begin{aligned}
& \sin (2 \pi k)\left[16 J_{3} \cos ^{3}(2 \pi k)+12 J_{2} \cos ^{2}(2 \pi k)\right. \\
& \left.\quad+\left(2 J_{1}^{\prime}-4 J_{3}\right) \cos (2 \pi k)+J_{1}-3 J_{2} \pm J_{4}\right]=0,
\end{aligned}
$$

which vanishes when $\sin (2 \pi k)=0$, i.e., $k=0$ or $k=$ $0.5(2 \pi k \in[0,2 \pi[)$ or when

$$
\begin{aligned}
& 16 J_{3} \cos ^{3}(2 \pi k)+12 J_{2} \cos ^{2}(2 \pi k)+\left(2 J_{1}^{\prime}-4 J_{3}\right) \cos (2 \pi k) \\
& \quad+J_{1}-3 J_{2} \pm J_{4}=0
\end{aligned}
$$

of the general form

$$
\alpha x^{3}+\beta x^{2}+\gamma x+\delta=0,
$$

with $x=\cos (2 \pi k), \alpha=16 J_{3}, \beta=12 J_{2}, \gamma=2 J_{1}^{\prime}-4 J_{3}$, and $\delta=J_{1}-3 J_{2} \pm J_{4}$. To solve such a third-degree equation, one should calculate its discriminant $\Delta$ according to

$$
\Delta=\frac{\Delta_{1}^{2}-4 \Delta_{0}^{3}}{-27 \alpha^{2}}
$$

where

$$
\Delta_{0}=\beta^{2}-3 \alpha \gamma
$$

and

$$
\Delta_{1}=2 \beta^{3}-9 \alpha \beta \gamma+27 \alpha^{2} \delta .
$$

The solutions of Eq. (5) depend on $\Delta$ such that we have three real solutions if $\Delta>0$, given by

$$
x_{\ell}=2 \sqrt{\frac{\Delta_{0}}{9 \alpha^{2}}} \cos \left[\frac{1}{3} \operatorname{arcos}\left(\frac{\Delta_{1}}{-6 \alpha \Delta_{0}} \sqrt{\frac{9 \alpha^{2}}{\Delta_{0}}}\right)-\ell \frac{2 \pi}{3}\right]-\frac{\beta}{3 \alpha},
$$

with $\ell=0,1,2$; two real solutions if $\Delta=0$, such that (i) if $\Delta_{0}=0$, then

$$
x_{1}=x_{2}=-\frac{\beta}{3 \alpha}
$$

and (ii) if $\Delta_{0} \neq 0$, then

$$
x_{1}=\frac{9 \alpha \delta-\beta \gamma}{2 \Delta_{0}}
$$

and

$$
x_{2}=\frac{4 \alpha \beta \gamma-9 \alpha^{2} \delta-\beta^{3}}{\alpha \Delta_{0}} ;
$$

and a unique real solution if $\Delta<0$, given by

$$
x=-\frac{1}{3 \alpha}\left(\beta+u+\frac{\Delta_{0}}{u}\right),
$$

with $u=\sqrt[3]{\frac{\Delta_{1}+\sqrt{-27 \alpha^{2} \Delta}}{2}}$. Therefore, the solutions of Eq. (3), which minimize the energy of Eq. (2), give the theoretical values of the propagation vector $\mathbf{q}$ in the infinite crystal of $\mathrm{CuCrO}_{2}$ and similar systems.

Thus, comparing the obtained values of $\mathbf{q}$ with that of neutron diffraction experiments allows proposing a realistic set of competing exchange interactions that leads to noncollinear magnetic ordering in frustrated systems.

\section{RESULTS AND DISCUSSION}

\section{A. DFT calculations}

To extract the various competing interactions that lead to the experimental propagation vector $\mathbf{q}=(0.329,0.329$, 0 ), we perform $\mathrm{DFT}+U[26,27]$ calculations in the distorted crystal structure of $\mathrm{CuCrO}_{2}$. Exchange interactions up to fourth-nearest neighbors are considered (Fig. 1). Since noncollinear DFT $+U$ calculations for the actual GS would be extremely hard and computationally demanding, exchange interactions are extracted from the collinear ferromagnetic 
TABLE I. Estimated DFT values of the exchange interactions (in $\mathrm{meV}$ ) for different choices of $U$ and $J_{H}$ in the undistorted structure $\left(d=0\right.$, i.e., $\left.J_{1}=J_{1}^{\prime}\right)$ of $\mathrm{CuCrO}_{2}$.

\begin{tabular}{lccccc}
\hline \hline$U(\mathrm{eV})$ & $J_{H}(\mathrm{eV})$ & $J_{1}$ & $J_{2}$ & $J_{3}$ & $J_{4}$ \\
\hline 2.3 & 0.96 & -2.407 & 0.012 & -0.266 & -0.060 \\
2.3 & 0.88 & -2.237 & 0.012 & -0.266 & -0.060 \\
3.0 & 0.96 & -1.681 & 0.012 & -0.242 & -0.048 \\
3.0 & 0.88 & -1.536 & 0.018 & -0.230 & -0.048 \\
\hline \hline
\end{tabular}

state. We have previously shown that the extracted values of exchange interactions are relatively robust and do not depend on the considered reference state [23]. Using constraint DFT calculations, Mazin estimated the values of Hubbard $U=$ $2.3 \mathrm{eV}$ and Hund's exchange $J_{H}=0.96 \mathrm{eV}$ for a similar system, $\mathrm{LiCrO}_{2}$ [28]. In order to know which values of $U$ and $J_{H}$ are the best for estimating the exchange interactions in $\mathrm{CuCrO}_{2}$, we test the estimates from Ref. [28] and other values of $U$ and $J_{H}$. For simplicity, we extract the exchange interactions in the undistorted structure $\left(d=0\right.$, i.e., $\left.J_{1}=J_{1}^{\prime}\right)$ as reported in Table I.

As one can see, $J_{1}$ is the most sensitive interaction relative to the others, and it is inversely proportional to the value of $U$, as can be expected for a superexchange coupling in dielectric systems. The interactions with more distant neighbors turn out to be extremely robust. On a qualitative level, the sign of exchange interactions remains the same regardless of the considered values of $U$ and $J_{H}$. Interestingly, we find that $U=$ $2.3 \mathrm{eV}$ gives $J_{1}$ in quite good agreement with experimental estimates [22,29]. Thus, the values of $U$ and $J_{H}$ are adopted from the work of Mazin [28] in further calculations.

Now, in the distorted crystal, several values of lattice distortion $d=\left(a-a^{\prime}\right) / a^{\prime}$ are examined. The $a$ parameter is set to the experimental lattice constant $(2.9746 \AA)$ and is kept fixed in the calculations, while $a^{\prime}$ is varied in such a way that it remains smaller than $a$. $J_{1}^{\prime}$ corresponds to the shorter distance to the neighboring spins (Fig. 1). The details of these calculations are the same as in our previous study [23]. Table II gives the estimated values of the exchange interactions for each value of $d$. It is worth noting that the magnitude of $J_{3}$ is much larger than that of $J_{2}$. This is a very nice effect, which can be explained by inspecting the corresponding exchange paths involving different $\mathrm{Cr} d$ states. As one can see in Fig. 2, even though the distance between the spins is smaller for the $J_{2}$ coupling compared to that of $J_{3}$, the exchange path is more indirect since it involves a virtual transition from one $\mathrm{Cr} d$ orbital to another on the

TABLE II. DFT estimates of exchange interactions (in $\mathrm{meV}$ ) for various distortions in $\mathrm{CuCrO}_{2}$.

\begin{tabular}{lccccc}
\hline \hline$d$ & $J_{1}^{\prime}$ & $J_{1}$ & $J_{2}$ & $J_{3}$ & $J_{4}$ \\
\hline 0 & -2.407 & -2.407 & 0.012 & -0.266 & -0.060 \\
0.0001 & -2.419 & -2.407 & 0.012 & -0.266 & -0.060 \\
0.001 & -2.516 & -2.395 & 0.012 & -0.266 & -0.060 \\
0.002 & -2.612 & -2.395 & 0.012 & -0.266 & -0.060 \\
0.003 & -2.709 & -2.383 & 0.012 & -0.266 & -0.060 \\
\hline \hline
\end{tabular}
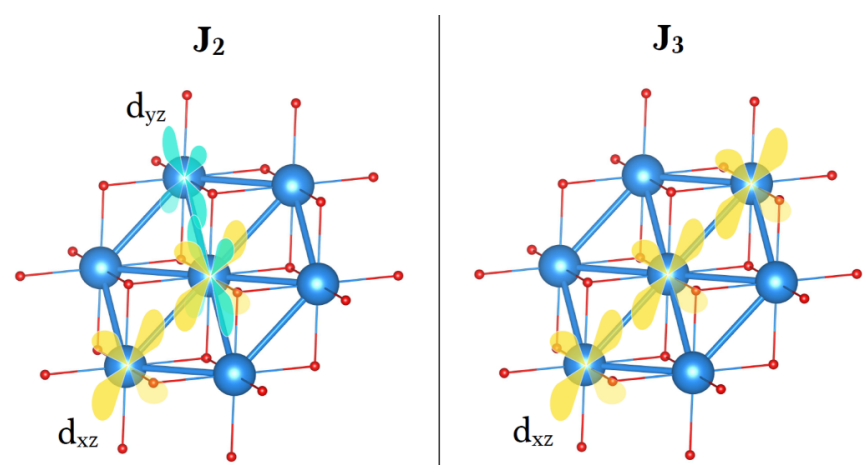

FIG. 2. Schematic representation of the exchange paths corresponding to the $J_{2}$ and $J_{3}$ interactions.

bridging $\mathrm{Cr}$ site. This can be viewed as a particular example of a $90^{\circ}$ superexchange, which is always small [30]. At the same time, $J_{3}$ coupling can be formed by hybridizing three $\mathrm{Cr}$ $d$ orbitals on the nearest-neighbor sites (directly or via $\mathrm{O} p$ states), which should provide a more efficient and substantial exchange, which is exactly what we see in our calculations.

\section{B. Theoretical values of the propagation vector}

The variation of $E_{\mathrm{ex}}^{\infty}$ [Eq. (2)] versus $k$ in the 3D infinite crystal is plotted in Fig. 3 for $d=0.001,0.002$, and 0.003 . It can be seen that each $E_{\mathrm{ex}}^{\infty}(k)$ curve possesses a single minimum corresponding to the unique real solution given by Eq. (13) (the case of $\Delta<0$ ) and two maxima at $k=0$ and 0.5 , corresponding to the solution of Eq. (3) for $\sin (2 \pi k)=0$. The theoretical values of $k$ corresponding to each minimum in the $E_{\mathrm{ex}}^{\infty}(k)$ curves are reported in Table III for various distortions. From a first observation, it can be seen that both an interlayer coupling and a lattice distortion slightly decrease the value of $k$. Starting with the undistorted two-dimensional (2D) infinite crystal $\left(J_{4}=0\right)$, we can clearly see that the GS configuration is a perfect $120^{\circ}(k=1 / 3)$. However, a tiny distortion $d=0.0001$ destabilizes the perfect $120^{\circ}$ configuration, leading to either an incommensurate or a commensurate magnetic

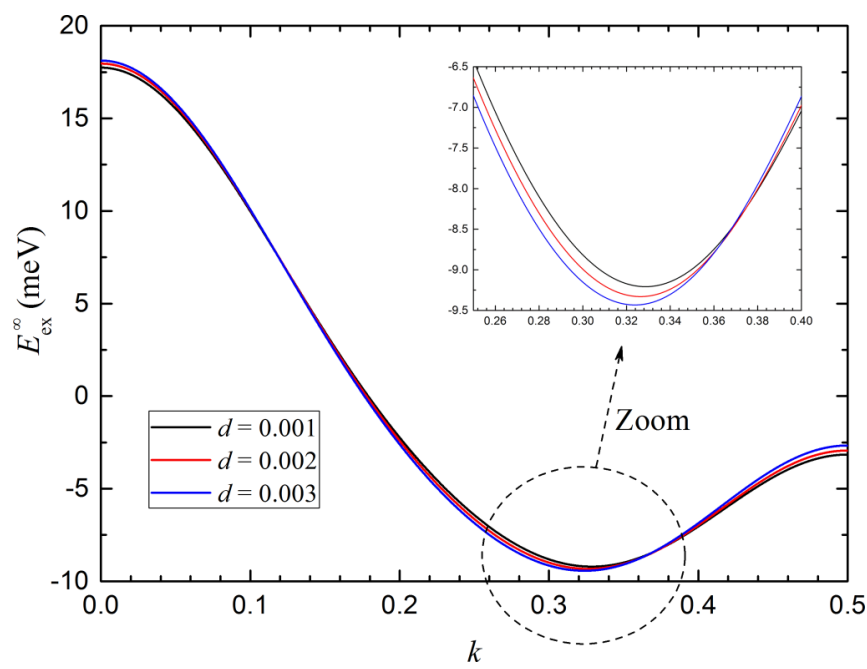

FIG. 3. Variation of $E_{\mathrm{ex}}^{\infty}$ versus $k$ for various distortions in the 3D infinite crystal of $\mathrm{CuCrO}_{2}$. 
TABLE III. Theoretical values of $\mathbf{q}=(k, k, 0)$ in the infinite 2D $\left(J_{4}=0\right)$ and $3 \mathrm{D}\left(J_{4} \neq 0\right)$ crystals calculated for various values of lattice distortion in $\mathrm{CuCrO}_{2}$.

\begin{tabular}{cccccc}
\hline \hline & \multicolumn{5}{c}{$d$} \\
\cline { 2 - 6 } & 0 & 0.0001 & 0.001 & 0.002 & 0.003 \\
\hline$k, 2 \mathrm{D}$ & 0.3333 & 0.3330 & 0.3303 & 0.3279 & 0.3254 \\
$k, 3 \mathrm{D}$ & 0.3318 & 0.3314 & 0.3287 & 0.3264 & 0.3238 \\
\hline \hline
\end{tabular}

configuration. In the undistorted 3D infinite crystal $\left(J_{4} \neq 0\right)$, the $120^{\circ}$ GS configuration does not exist $(k<1 / 3)$, indicating that a very weak interlayer coupling can destabilize this perfect $120^{\circ}$ state. Considering the experimental distortion $d=$ 0.0001 estimated at $T=5 \mathrm{~K}$ [21], it can clearly be seen that the theoretical value of $k$ in the infinite crystal is slightly larger than that reported experimentally $(k=0.329)$. Thus, we can say that, within the framework of the classical Heisenberg model, $d=0.0001$ cannot describe the experimental GS even in the infinite crystal. Interestingly, a $0.1 \%$ lattice distortion yields $\mathbf{q} \sim(0.329,0.329,0)$ in the $3 \mathrm{D}$ infinite crystal of $\mathrm{CuCrO}_{2}$. The dominant exchange parameters corresponding to $d=0.1 \%$ are very comparable to those obtained from the inelastic neutron scattering experiment, as shown in Table IV. The main difference between our DFT estimates and inelastic neutron scattering ones is seen mainly in the next-nearestneighbor $J_{2}$, concerning both its nature and magnitude.

\section{Effects of the nature and magnitudes of next-nearest-neighbor interactions on the ground state of $\mathrm{CuCrO}_{2}$}

It is clear that the first-nearest-neighbor interactions $J_{1}$ and $J_{1}^{\prime}$ are antiferromagnetic (AFM) in this crystal. Their order of magnitude is found to be similar in various theoretical $[23,31]$ and experimental $[22,29,32]$ studies, ranging between -2.97 and $-2.30 \mathrm{meV}$. However, a lack of precision covers the nextnearest-neighbor interactions $J_{2}, J_{3}$, and $J_{4}$, concerning their nature due to their small magnitudes. The case of $J_{3}$ is less confused. This work and previous studies $[23,31,32]$ suggest that $J_{3}$ is an AFM coupling varying between -0.27 and $-0.08 \mathrm{meV}$. However, contradictory results are reported for $J_{2}$ and $J_{4}$. This work and previous DFT calculations [23,31] show that $J_{2}$ is ferromagnetic (FM), while neutron scattering measurements [29,32] find an AFM $J_{2}$ coupling. The reverse scenario is reported for $J_{4}$.

Therefore, we investigate here the stable magnetic configuration taking into account both possible signs (FM and AFM) of $J_{2}, J_{3}$, and $J_{4}$ using the derived analytical expression of $\mathbf{q}$ (Sec II). Based on that, we determine the nature and order

TABLE IV. Comparison of our DFT estimates of exchange interactions (in $\mathrm{meV}$ ) for $d=0.1 \%$ with those obtained from inelastic neutron scattering measurements in $\mathrm{CuCrO}_{2}$.

\begin{tabular}{lccccc}
\hline \hline Study & $J_{1}^{\prime}$ & $J_{1}$ & $J_{2}$ & $J_{3}$ & $J_{4}$ \\
\hline This work & -2.516 & -2.395 & 0.012 & -0.266 & -0.060 \\
Ref. [29] & -2.53 & -2.3 & -0.12 & & \\
\hline \hline
\end{tabular}

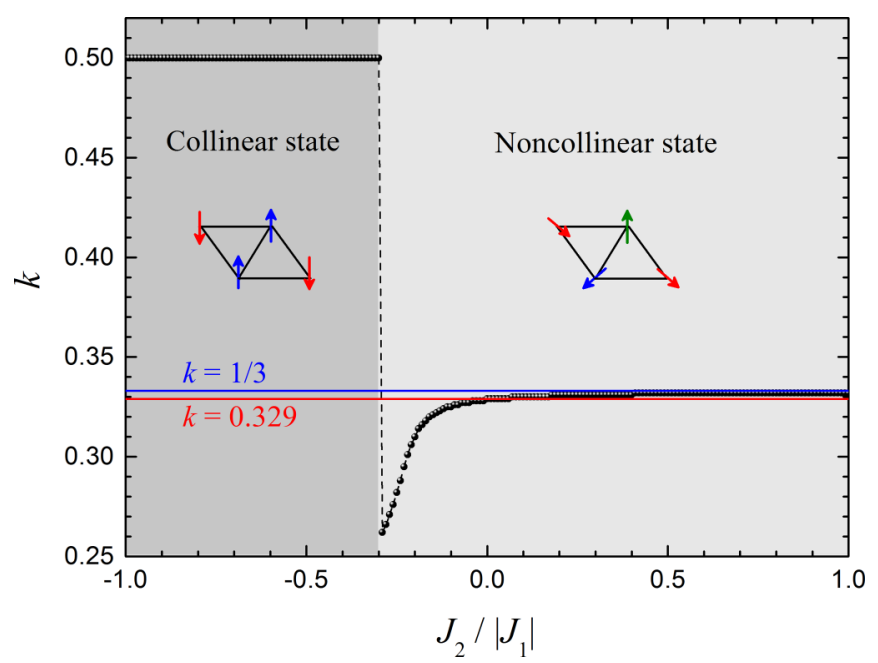

FIG. 4. Variation of the theoretical value of $k$ versus $J_{2} /\left|J_{1}\right|$ for $J_{1}, J_{1}^{\prime}, J_{3}$, and $J_{4}$ corresponding to $d=0.001$.

of magnitudes of the next-nearest-neighbor interactions which stabilize the experimental noncollinear $\mathbf{q}=(0.329,0.329,0)$ magnetic configuration in $\mathrm{CuCrO}_{2}$.

\section{Effect of $J_{2}\left(-1 \leqslant J_{2} /\left|J_{1}\right| \leqslant 1\right)$}

Here we examine the effect of having FM or AFM $J_{2}$ on the magnetic ordering in $\mathrm{CuCrO}_{2}$. For that we calculate $\mathbf{q}=$ $(k, k, 0)$ for each value of $J_{2} /\left|J_{1}\right|$ in the interval $[-1,1]$. The dependence of $k$ on $J_{2} /\left|J_{1}\right|$ is plotted in Fig. 4. It can be seen that the noncollinear state is stabilized for every FM $J_{2}$ with $0.329 \leqslant k \leqslant 0.332$. However, for AFM $J_{2}$, the noncollinear state exists only for $-0.299 \leqslant J_{2} /\left|J_{1}\right| \leqslant 0$, while it becomes a collinear state for $J_{2} /\left|J_{1}\right| \leqslant-0.3$. Interestingly, the $\mathbf{q}=$ $(0.329,0.329,0)$ configuration is stabilized only if $J_{2} /\left|J_{1}\right| \in$ $[-0.007,0.053]$, where our DFT estimate of $J_{2}$ lies.

\section{Effect of $J_{3}\left(-1 \leqslant J_{3} /\left|J_{1}\right| \leqslant 1\right)$}

Following the same investigation as for $J_{2}$, the dependence of $k$ on $J_{3} /\left|J_{1}\right| \in[-1,1]$ is given in Fig. 5. It is clear that an AFM $J_{3}$ is favorable for AFM noncollinear magnetic ordering in $\mathrm{CuCrO}_{2}$. FM $J_{3}$ favors AFM collinear magnetic ordering with $k=0.5$ starting from $J_{3} /\left|J_{1}\right|=0.14$ and above. Interestingly, the $\mathbf{q}=(0.329,0.329,0)$ configuration is preserved when $J_{3} /\left|J_{1}\right| \in[-0.186,-0.091]$, where our DFT estimate of $J_{3}$ satisfies this condition.

$$
\text { 3. Effect of } J_{4}\left(-1 \leqslant J_{4} /\left|J_{1}\right| \leqslant 1\right)
$$

A different scenario is observed for $J_{4}$. Noncollinear magnetic ordering exists for any value of $J_{4} /\left|J_{1}\right| \in[-1,1]$, as shown in Fig. 6. Whatever the sign of $J_{4}$ is, $k$ decreases when $\left|J_{4}\right|$ increases. The same value of $k$ is obtained for a given magnitude of $J_{4}$ whether $J_{4}$ is FM or AFM. However, the $\mathbf{q}=(0.329,0.329,0)$ configuration is stabilized for AFM interlayer coupling when $J_{4} /\left|J_{1}\right| \in[-0.029,-0.014]$, where our DFT estimate of $J_{4}$ lies, or FM interlayer coupling when $J_{4} /\left|J_{1}\right| \in[0.014,0.029]$. 


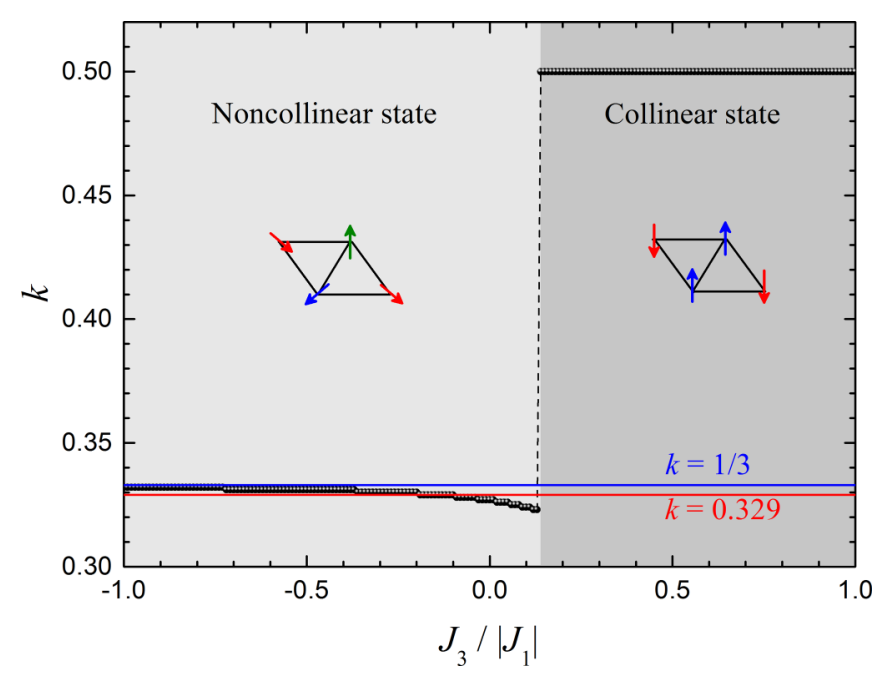

FIG. 5. Variation of the theoretical value of $k$ versus $J_{3} /\left|J_{1}\right|$ for $J_{1}, J_{1}^{\prime}, J_{2}$, and $J_{4}$ corresponding to $d=0.001$.

\section{Monte Carlo simulations}

In order to validate the GS predicted by our analytical calculation of $k$ in the 3D infinite crystal with $d=0.001$, we perform a simulated annealing using the Monte Carlo (MC) method [33]. A finite 3D crystal of $\mathrm{CuCrO}_{2}$ of size $L \times L \times L_{z}$ is built with only the magnetic $\mathrm{Cr}^{3+}(S=3 / 2)$ ions. However, simulating noncollinear magnetic configurations derived from high geometric magnetic frustration is not straightforward. Indeed, noncollinear magnetic ordering (GS and phase transition) in finite systems is affected by size and boundary effects, where the stable magnetic configuration can be different from that of the infinite system. Two different cases should be distinguished: (i) The GS is commensurate, and then it is possible to find a size $L$ such that $(L+1)$ is a multiple of the magnetic period and hence periodic boundary conditions (PBCs) are suitable for simulating the GS. (ii) The GS is incommensurate; that is, it is not periodic, and thus, PBCs (as well as free boundary conditions) will significantly perturb

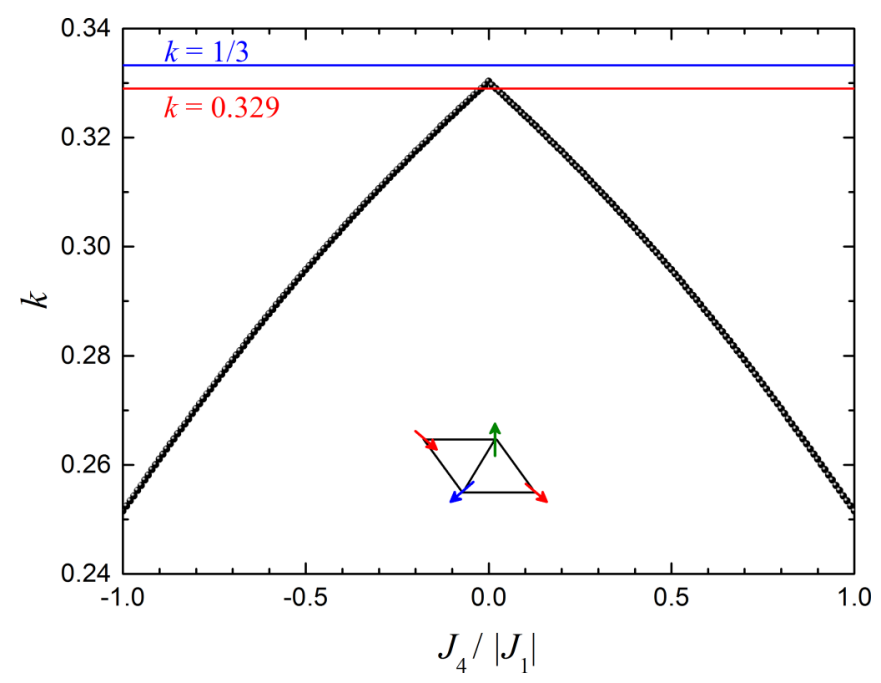

FIG. 6. Variation of the theoretical value of $k$ versus $J_{4} /\left|J_{1}\right|$ for $J_{1}, J_{1}^{\prime}, J_{2}$, and $J_{3}$ corresponding to $d=0.001$. the simulated GS if the system size $L$ is wrongly chosen. Therefore, to find the system sizes that are able to reproduce the true incommensurate GS magnetic configurations, we calculate analytically the exchange energy per spin of a finite magnetic configuration with a propagation vector $\mathbf{q}=(k, k, 0)$ on a rhombohedrally stacked distorted triangular lattice, using PBCs, as a function of $k, L$, and $L_{z}$. Based on Eq. (1), we find

$$
\begin{aligned}
& E_{1}(k, L)=-\frac{1}{2} S^{2} \frac{1}{L^{2}}\left\{4 J_{1} L(L-1) \cos [2 \pi k]\right. \\
& +2(L-1)^{2} J_{1}^{\prime} \cos [4 \pi k]+4 L J_{1} \cos [2 \pi k(L-1)] \\
& +4(L-1) J_{1}^{\prime} \cos [2 \pi k(L-2)] \\
& \left.+2 J_{1}^{\prime} \cos [4 \pi k(L-1)]\right\} \text {, } \\
& E_{2}(k, L)=-\frac{1}{2} J_{2} S^{2} \frac{1}{L^{2}}\left\{2 L^{2}-4 L+23+\cos [2 \pi k]\right. \\
& +4\left(L^{2}-3 L+8\right) \cos [6 \pi k] \\
& +\cos [4 \pi k(L-1)]+3 \cos [2 \pi k(L+1)] \\
& +2(L-3) \cos [2 \pi k(L-1)] \\
& +(4 L-5) \cos [2 \pi k L]+5 \cos [2 \pi k(2 L-3)] \\
& +2 \cos [2 \pi k(L-2)]+2 \cos [2 \pi k(L+2)] \\
& +10(L-2) \cos [2 \pi k(L-3)] \\
& +3 \cos [2 \pi k(L+3)]\} \text {, } \\
& E_{3}(k, L)=-\frac{1}{2} J_{3} S^{2} \frac{1}{L^{2}}\{4 L(L-2) \cos [4 \pi k] \\
& +2(L-2)^{2} \cos [8 \pi k]+8 L \cos [2 \pi k(L-2)] \\
& +8(L-2) \cos [2 \pi k(L-4)] \\
& +8 \cos [4 \pi k(L-2)]\} \text {, } \\
& E_{4}\left(k, L, L_{z}\right)= \pm \frac{1}{2} J_{4} S^{2} \frac{1}{3 L^{2} L_{z}}\left\{6 L_{z} L^{2}+\left(-4 L^{2}+12 L_{z} L^{2}\right.\right. \\
& \left.+2 L-9 L_{z} L-1\right) \cos [2 \pi k] \\
& +\left(4 L^{2}-L\right) \cos \left[2 \pi k\left(3 L_{z}-1\right)\right] \\
& +\left(6 L_{z} L+6 L_{z}-4\right) \cos [2 \pi k(L-1)] \\
& \left.+(L+2) \cos \left[2 \pi k(L-1)\left(3 L_{z}-1\right)\right]\right\},
\end{aligned}
$$

and hence

$$
\begin{aligned}
E_{\mathrm{ex}}\left(k, L, L_{z}\right)= & E_{1}(k, L)+E_{2}(k, L) \\
& +E_{3}(k, L)+E_{4}\left(k, L, L_{z}\right) .
\end{aligned}
$$

Note that Eq. (18) leads to Eq. (2) when $L$ and $L_{z}$ tend to infinity. Figure 7 shows the variation of the exchange energy per spin [Eq. (18)] of a magnetic configuration with $k \simeq 0.329$ versus $L$ for a given $L_{z}$. A strong $L$ dependence of the exchange energy is seen. For example, $E_{\mathrm{ex}}=-8.927 \mathrm{meV}$ for $L=72$ is higher than that of the infinite system $E_{\mathrm{ex}}^{\infty}=$ $-9.208 \mathrm{meV}$ because the magnetic configuration of $k=$ 0.329 does not match the box size well. However, for $L=73$, $E_{\mathrm{ex}}=-9.208 \mathrm{meV}$ coincides with $E_{\mathrm{ex}}^{\infty}$, meaning that the magnetic configuration with $k=0.329$ does match with this box size well. Thus, only sizes that have $E_{\mathrm{ex}}\left(k, L, L_{z}\right)$ very close to that of the infinite crystal should be considered in 


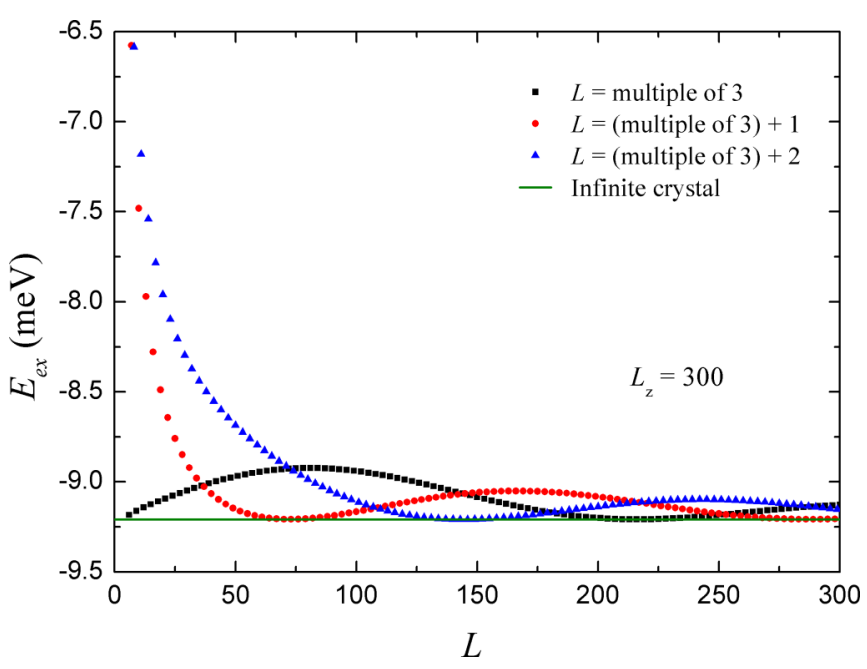

FIG. 7. $L$ dependence of the exchange energy per spin of a configuration with $\mathbf{q} \sim(0.329,0.329,0)$ calculated with the set of exchange interactions corresponding to $d=0.001$.

numerical simulations. To elucidate boundary and finite-size effects on the propagation vector, we study the $k$ dependence of $E_{\text {ex }}$ for both sizes $L=72$ and $L=73$ in comparison with that in the infinite crystal, as shown in Fig. 8. It can be seen that finite-size effects (oscillations) exist in both cases. However, the choice of $L$ (and thus boundary effects) can completely change the magnetic ordering of the system. It can be seen that the minimum of $E_{\mathrm{ex}}$ for $L=73$ coincides with that of the infinite crystal at $k=0.3287$, preserving the incommensurate nature of the magnetic configuration. However, for $L=72$, the stable magnetic configuration is close to the perfect $120^{\circ}$ with $k=0.3334$ because PBCs impose this ordering.

Note that for a given $L$ that has $E_{\mathrm{ex}}\left(k, L, L_{z}\right)$ very close to that of the infinite crystal, the dependence of $E_{\mathrm{ex}}\left(k, L, L_{z}\right)$ on $L_{z}$ is much less pronounced (not shown here) due to the small value of $J_{4}$.

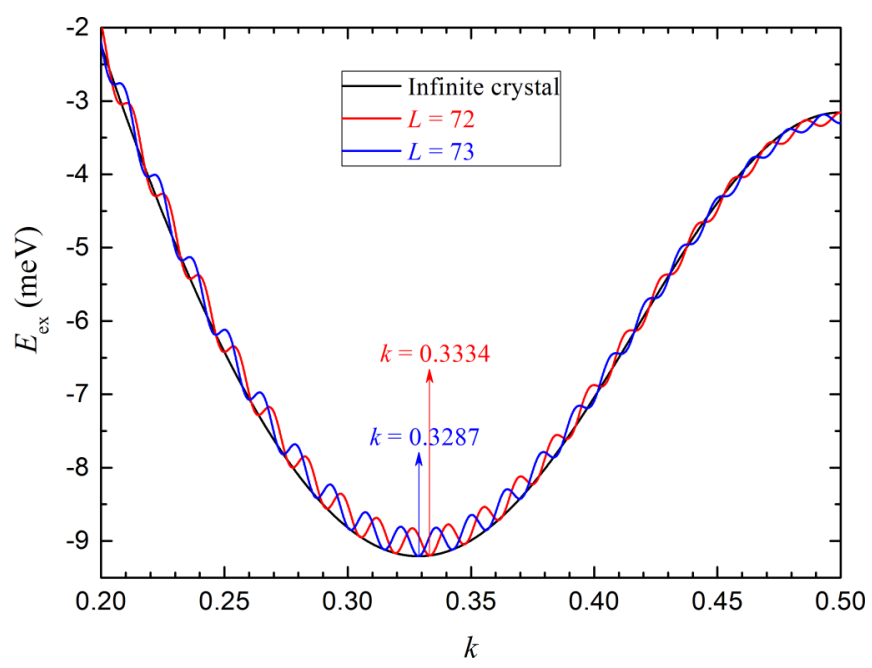

FIG. 8. The $k$ dependence of the exchange energy per spin in finite crystals compared to that in the infinite crystal calculated with the set of exchange interactions corresponding to $d=0.001$.

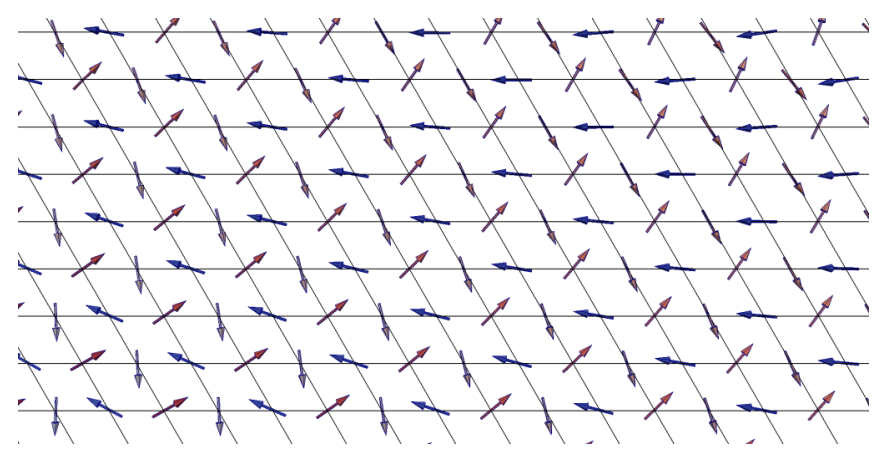

FIG. 9. Simulated ground-state magnetic configuration with $\mathbf{q}=$ $(0.3288,0.3288,0)$ in $\mathrm{CuCrO}_{2}$.

Based on the previous analyses, we simulate a finite crystal of size $73 \times 73 \times 2$ using PBCs to validate the predicted GS based on the Heisenberg Hamiltonian given in Eq. (1). The set of exchange interactions corresponding to $d=0.001$ is considered (Table II).

Our MC simulations are performed using the standard Metropolis algorithm [34]. We start our simulations from random spin configurations at a high enough temperature $T_{i}=35.01 \mathrm{~K}$ above the transition temperature of the system. We then cool the system down to a final temperature $T_{f}=$ $0.01 \mathrm{~K}$ according to $T_{i+1}=T_{i}-\Delta T$. At each temperature, we perform $55 \times 10^{3} \mathrm{MC}$ steps, where the first $5 \times 10^{3} \mathrm{MC}$ steps are discarded for thermal equilibration.

We find that the magnetic system undergoes a phase transition from a paramagnetic state into an antiferromagnetic noncollinear state at Néel temperature $T_{N}=27.5 \pm 0.5 \mathrm{~K}$, which agrees well with the ones reported experimentally. This would suggest the ability of our DFT estimates of exchange interactions to describe the various magnetic properties of $\mathrm{CuCrO}_{2}$.

At $T_{f}$, we find that the simulated energy $E_{\mathrm{ex}}^{\text {sim }} \approx$ $-9.203 \mathrm{meV}$ is in excellent agreement with that calculated analytically, $E_{\text {ex }}(k=0.329) \approx-9.202 \mathrm{meV}$ [Eq. (18) for $L=73$ and $L_{z}=2$ ], indicating that the simulated GS is not perturbed by size or boundary effects.

Figure 9 shows the simulated GS magnetic configuration, where we can clearly see the various sublattices. For a precise characterization of this GS, we calculate the magnetic propagation vector $\mathbf{q}=(k, k, 0)$ according to

$$
k=\frac{1}{2 \pi} \operatorname{arcos}\left[\frac{\mathbf{S}_{i} \cdot \mathbf{S}_{j}}{S^{2}}\right],
$$

where the scalar product $\mathbf{S}_{i} \cdot \mathbf{S}_{j}$ is calculated for the spins along the [100] or [010] direction. Our simulations give a magnetic propagation vector $\mathbf{q}=(0.3288,0.3288,0)$, which reflects an incommensurate GS configuration $(2 \pi k=$ $118.37^{\circ}$ ) consistent with those calculated analytically and measured experimentally.

Furthermore, it is well known that spin chirality plays an essential role in quantifying magnetic ordering in frustrated systems. Thus, we consider the vector chirality per plane as an order parameter defined as

$$
\boldsymbol{\kappa}=\frac{1}{N_{p}} \frac{1}{S^{2}} \frac{2}{3 \sqrt{3}} \sum_{p}\left(\mathbf{S}_{1} \times \mathbf{S}_{2}+\mathbf{S}_{2} \times \mathbf{S}_{3}+\mathbf{S}_{3} \times \mathbf{S}_{1}\right),
$$




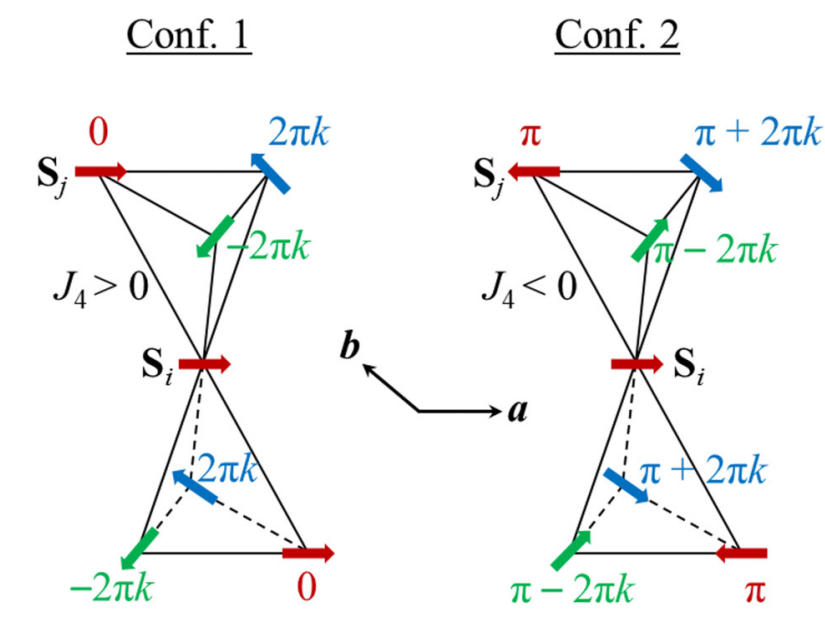

Conf. 3

Conf. 4

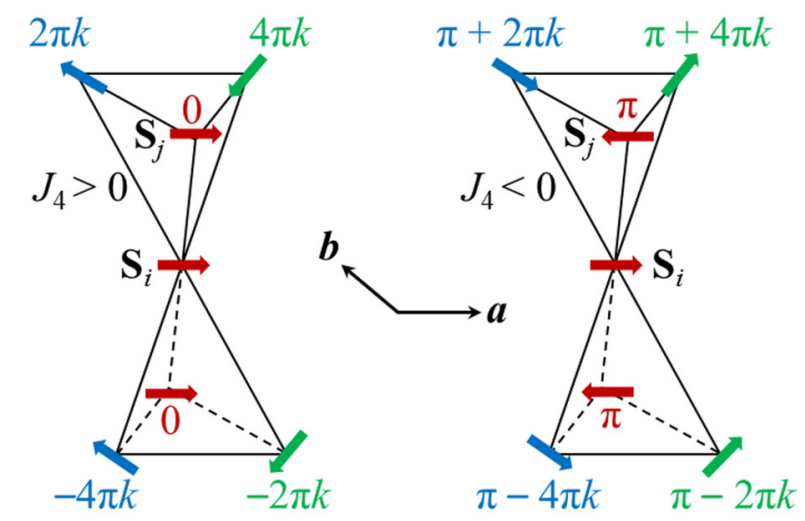

Conf. 5

$\underline{\text { Conf. } 6}$
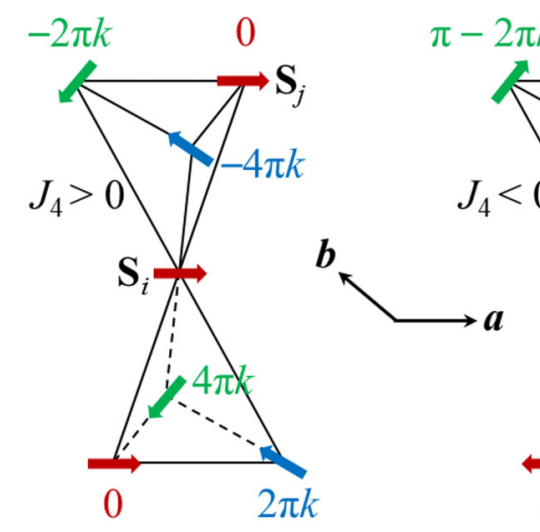

FIG. 10. Possible magnetic configurations with FM $\left(J_{4}>0\right)$ and $\operatorname{AFM}\left(J_{4}<0\right)$ interlayer couplings in $\mathrm{CuCrO}_{2}$.

where $N_{p}$ is the number of up plaquettes in each triangular layer; $\mathbf{S}_{1}, \mathbf{S}_{2}$, and $\mathbf{S}_{3}$ are the spins located at the corners of each elementary up plaquette (dashed plaquette in Fig. 1);

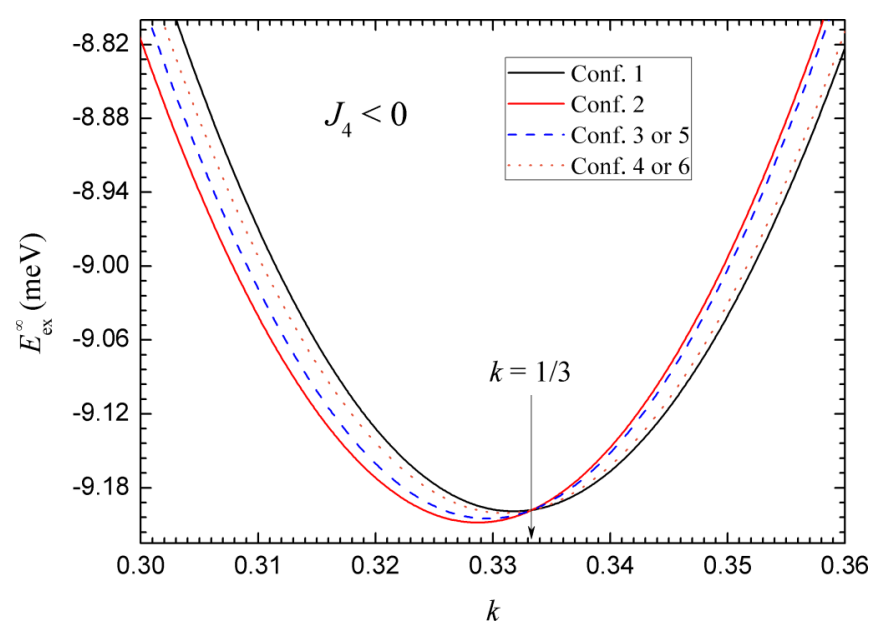

FIG. 11. $E_{\mathrm{ex}}^{\infty}$ (in meV) vs $k$ plot for the six possible configurations with $\mathrm{AFM}\left(J_{4}<0\right)$ interlayer couplings in $\mathrm{CuCrO}_{2}$.

and the sum runs over all up plaquettes in each layer. Note that $|\boldsymbol{\kappa}|=1$ in the perfect $120^{\circ}$ configuration, whereas $|\boldsymbol{\kappa}|$ is slightly smaller than 1 for $k$ close to $1 / 3$. At $T_{f}$, we find that $|\boldsymbol{\kappa}|=0.999$ within each triangular layer, which is in excellent agreement with its theoretical value calculated for $k=0.329$ according to

$$
|\kappa|_{\text {theo }}=\frac{2}{3 \sqrt{3}}[2 \sin (2 \pi k)-\sin (4 \pi k)]=0.999,
$$

confirming the simulated value of $\mathbf{q}$ at the GS. Such good convergence of our MC simulations toward the true GS in a 3D infinite crystal would confirm the validity of our derived analytical expressions of $k$ and those of the exchange energy in 3D finite crystals [Eqs. (14)-(17)], which serves as an important presimulation step to warrant the convergence of simulations toward theory.

\section{CONCLUSIONS}

In conclusion, a compact analytical model that calculates the magnetic propagation vector as a function of exchange interactions up to fourth-nearest neighbors in rhombohedrally stacked distorted triangular lattices was proposed. The ingredients of this model are calculated from first principles using $\mathrm{DFT}+U$ calculations. Within our model, we found that the set of exchange interactions resulting from a $0.1 \%$ lattice distortion reproduces the experimental ground state with a propagation vector $\mathbf{q} \sim(0.329,0.329,0)$ in the distorted infinite crystal of $\mathrm{CuCrO}_{2}$. Also, our results suggested that a ferromagnetic next-nearest-neighbor interaction $J_{2}$ and a very weak antiferromagnetic interlayer interaction $J_{4}$ are favorable for stabilizing the $\mathbf{q}=(0.329,0.329,0)$ configuration in $\mathrm{CuCrO}_{2}$. Finally, Monte Carlo simulations performed in $\mathrm{CuCrO}_{2}$ converge well toward the experimental ground state, validating our analytical and $\mathrm{DFT}+U$ calculations.

\section{ACKNOWLEDGMENTS}

This work was supported by the French Agence Nationale de la Recherche and LabEx EMC3 through the Project AFLTEPMAG (Grant No. ANR-10-LABX-09-01). The 
TABLE V. GS configuration (Conf.) as a function of the nature of $J_{4}$ and the value of $k$ in $\mathrm{CuCrO}_{2}$.

\begin{tabular}{lll}
\hline \hline & $k<1 / 3$ & $k>1 / 3$ \\
\hline$J_{4}<0$ & Conf. 2 & Conf. 1 \\
$J_{4}>0$ & Conf. 1 & Conf. 2 \\
\hline \hline
\end{tabular}

authors acknowledge the computational resources provided by the Swedish National Infrastructure for Computing (SNIC) and Uppsala Multidisciplinary Center for Advanced Computational Science (UPPMAX). Monte Carlo simulations were carried out on the supercomputer of the Centre Régional Informatique et d'Applications Numériques de Normandie (CRIANN) as Project No. 2015004.

\section{APPENDIX}

\section{Minimal-energy configuration with interlayer couplings}

In the presence of $J_{4}$, six magnetic configurations, shown in Fig. 10, are possible at the GS. Their energies are $E_{4}=$ $-J_{4} S^{2}[1+2 \cos (2 \pi k)]$ for configuration $1, E_{4}=+J_{4} S^{2}[1+$ $2 \cos (2 \pi k)]$ for configuration $2, E_{4}=-J_{4} S^{2}[1+\cos (2 \pi k)+$ $\cos (4 \pi k)]$ for configurations 3 and 5 , and $E_{4}=+J_{4} S^{2}$ $[1+\cos (2 \pi k)+\cos (4 \pi k)]$ for configurations 4 and 6 . Generally, the stable configuration is determined by both the nature of $J_{4}$ (FM or AFM) and the value of $k$. For example, if $J_{4}<0$, configuration 2 is stable for $k<1 / 3$, while configuration 1 becomes more stable when $k>1 / 3$, as seen in Fig. 11 .
TABLE VI. DFT estimates of the single-ion anisotropy constants (in $\mathrm{meV}$ ) in $\mathrm{CuCrO}_{2}$.

\begin{tabular}{lccccr}
\hline \hline$d$ & 0 & 0.0001 & 0.001 & 0.002 & 0.003 \\
\hline$D_{x}$ & 0 & 0 & -0.0004 & -0.0007 & -0.001 \\
$D_{z}$ & 0.033 & 0.033 & 0.033 & 0.033 & 0.033 \\
\hline \hline
\end{tabular}

However, for $J_{4}>0$, the case is the opposite, as summarized in Table V.

\section{Anisotropy}

It is important to note that the magnetocrystalline anisotropy is calculated in this work for each value of the considered distortions. The DFT estimates of the single-ion anisotropy constants $D_{x}$ and $D_{z}$ corresponding to hard and easy axes along the [110] and [001] directions, respectively, are listed in Table VI. As can be noticed, lattice distortion induces the hard-axis anisotropy in $\mathrm{CuCrO}_{2}$, resulting in an easy-plane anisotropy, the $y z$ plane.

Nonetheless, magnetocrystalline anisotropy was not introduced in the previous analytical calculations of $\mathbf{q}$ because it does not affect the magnetic ordering in the system [35]. For validity, the same previous MC simulations were repeated with the corresponding values of $D_{x}$ and $D_{z}$, where we obtained the same magnetic propagation vector $\mathbf{q}=(0.3288$, $0.3288,0)$ without any noticeable effect on the magnetic ordering of the system.
[1] M. F. Collins and O. A. Petrenko, Can. J. Phys. 75, 605 (1997).

[2] O. A. Starykh, W. Jin, and A. V. Chubukov, Phys. Rev. Lett. 113, 087204 (2014).

[3] O. A. Starykh, Rep. Prog. Phys. 78, 052502 (2015).

[4] D. Yamamoto, G. Marmorini, and I. Danshita, Phys. Rev. Lett. 114, 027201 (2015).

[5] R. D. Shannon, D. B. Rogers, and C. T. Prewitt, Inorg. Chem. 10, 713 (1971)

[6] C. T. Prewitt, R. D. Shannon, and D. B. Rogers, Inorg. Chem. 10, 719 (1971).

[7] D. B. Rogers, R. D. Shannon, C. T. Prewitt, and J. L. Gillson, Inorg. Chem. 10, 723 (1971).

[8] M. A. Marquardt, N. A. Ashmore, and D. P. Cann, Thin Solid Films 496, 146 (2006).

[9] R. Daou, R. Frésard, V. Eyert, S. Hébert, and A. Maignan, Sci. Technol. Adv. Mater. 18, 919 (2017).

[10] K. Kimura, H. Nakamura, K. Ohgushi, and T. Kimura, Phys. Rev. B 78, 140401(R) (2008).

[11] K. Kimura, H. Nakamura, S. Kimura, M. Hagiwara, and T. Kimura, Phys. Rev. Lett. 103, 107201 (2009).

[12] M. Soda, K. Kimura, T. Kimura, and K. Hirota, Phys. Rev. B 81, 100406(R) (2010).

[13] E. Mun, M. Frontzek, A. Podlesnyak, G. Ehlers, S. Barilo, S. V. Shiryaev, and V. S. Zapf, Phys. Rev. B 89, 054411 (2014).

[14] S.-Z. Lin, K. Barros, E. Mun, J.-W. Kim, M. Frontzek, S. Barilo, S. V. Shiryaev, V. S. Zapf, and C. D. Batista, Phys. Rev. B 89, 220405(R) (2014).
[15] Y. A. Sakhratov, L. E. Svistov, P. L. Kuhns, H. D. Zhou, and A. P. Reyes, Phys. Rev. B 94, 094410 (2016).

[16] A. Miyata, O. Portugall, D. Nakamura, K. Ohgushi, and S. Takeyama, Phys. Rev. B 96, 180401(R) (2017).

[17] J. P. Doumerc, A. Wichainchai, A. Ammar, M. Pouchard, and P. Hagenmuller, Mater. Res. Bull. 21, 745 (1986).

[18] T. Okuda, N. Jufuku, S. Hidaka, and N. Terada, Phys. Rev. B 72, 144403 (2005).

[19] S. Seki, Y. Onose, and Y. Tokura, Phys. Rev. Lett. 101, 067204 (2008).

[20] M. Poienar, F. Damay, C. Martin, V. Hardy, A. Maignan, and G. André, Phys. Rev. B 79, 014412 (2009).

[21] K. Kimura, T. Otani, H. Nakamura, Y. Wakabayashi, and T. Kimura, J. Phys. Soc. Jpn. 78, 113710 (2009).

[22] H. Yamaguchi, S. Ohtomo, S. Kimura, M. Hagiwara, K. Kimura, T. Kimura, T. Okuda, and K. Kindo, Phys. Rev. B 81, 033104 (2010).

[23] A. Albaalbaky, Y. O. Kvashnin, D. Ledue, R. Patte, and R. Frésard, Phys. Rev. B 96, 064431 (2017).

[24] N. Terada, J. Phys.: Condens. Matter 26, 453202 (2014).

[25] A. Albaalbaky, Y. O. Kvashnin, R. Patte, R. Frésard, and D. Ledue, Phys. Rev. B 98, 174403 (2018).

[26] M. T. Czyzyk and G. A. Sawatzky, Phys. Rev. B 49, 14211 (1994).

[27] A. I. Liechtenstein, V. I. Anisimov, and J. Zaanen, Phys. Rev. B 52, R5467(R) (1995). 
[28] I. I. Mazin, Phys. Rev. B 75, 094407 (2007).

[29] M. Poienar, F. Damay, C. Martin, J. Robert, and S. Petit, Phys. Rev. B 81, 104411 (2010).

[30] J. Kanamori, J. Phys. Chem. Solids 10, 87 (1959).

[31] X.-F. Jiang, X.-F. Liu, Y.-Z. Wu, and J.-R. Han, Chin. Phys. B 21, 077502 (2012).

[32] M. Frontzek, J. T. Haraldsen, A. Podlesnyak, M. Matsuda, A. D. Christianson, R. S. Fishman, A. S. Sefat, Y. Qiu, J. R. D.
Copley, S. Barilo, S. V. Shiryaev, and G. Ehlers, Phys. Rev. B 84, 094448 (2011).

[33] D. P. Landau and K. Binder, A Guide to Monte Carlo Simulations in Statistical Physics (Cambridge University Press, Cambridge, 2008).

[34] N. Metropolis, A. W. Rosenbluth, M. N. Rosenbluth, A. H. Teller, and E. Teller, J. Chem. Phys. 21, 1087 (1953).

[35] R. S. Fishman, J. Phys.: Condens. Matter 23, 366002 (2011). 